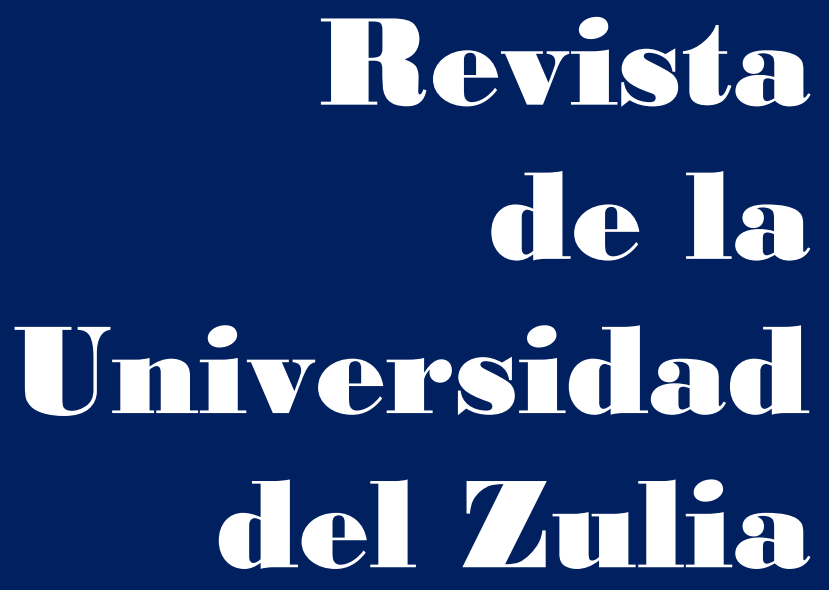

Fundada en 1947

por el Dr. Jesús Enrique Lossada

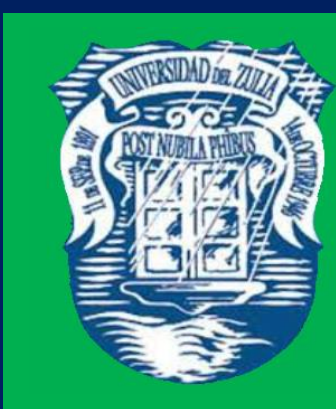

Ciencias del

Agred

Ingemieria

y Teenología
Añต 12 No 32

Enero - Abril 2021

Tercera Época

Maracailbo-Venezuela 
REVISTA DE LA UNIVERSIDAD DEL ZULIA. 3ª época. Año 12 N 32, 2021 Arnaldo Vergara-Romero et al. //La soberanía alimentaria en el desarrollo local, 54-69

\title{
La soberanía alimentaria en el desarrollo local
}

\author{
Arnaldo Vergara-Romero* \\ Rafael Sorhegui-Ortega** \\ César Salvador-Guerra***
}

RESUMEN

El presente artículo contiene una perspectiva de la Soberanía alimentaria desde el Desarrollo local, donde el primer concepto complementa al desarrollo territorial indistinto a la escala que se maneje. Es muy imperativo que para evaluar las potencialidades del desarrollo local se tiene que emplear como pilar, el derecho a la alimentación, ya que este radica un problema sustancial y evidente en la actualidad a nivel mundial, este problema es la deficiencia nutricional. También se analiza que este metabolismo social puede ayudar a buscar una solución desde lo local a lo regional e incluso a lo global, complementándose los dos conceptos para el beneficio de la sociedad. El tipo de metodología que se utilizó consistió en una revisión sistemática de información actualizada en artículos académicos sobre Soberanía alimentaria y Desarrollo local, la cual fue consultada en bases especializadas como Scopus y Web of Science.

PALABRAS CLAVE: bienes comunes, desarrollo local, desarrollo sostenible, desarrollo territorial, soberanía alimentaria.

*Affiliation: Universidad Ecotec and Universidad Espíritu Santo. MSc.in Economics, Universidad Ecotec and Universidad Espíritu Santo, Docente-Investigador, Research Departments, Samborondón, Ecuador. avergarar@ecotec.edu.ec, ORCID: https://orcid.org/0000-0001-8503-3685

**Affiliation: Universidad Ecotec. Ph.D. in Economics, Universidad Ecotec, Research Director, Research Departments, Samborondón, Ecuador. rsorhegui@ecotec.edu.ec. ORCID: https://orcid.org/0000-0001-7882-5246

*** Affiliation: Universidad Ecotec. Msc. in Economics, Universidad Ecotec, Egresado, Research Departments, Samborondón, Ecuador. cesalvador@delfos.edu.ec 
REVISTA DE LA UNIVERSIDAD DEL ZULIA. 3ª época. Año 12 N 32, 2021

\section{Food sovereignty in local development}

ABSTRACT

This article contains a perspective of Food Sovereignty from local development, where the first concept complements the indistinct territorial development at the scale that is managed. It is very imperative that to evaluate the potentialities of local development, the right to food must be used as a pillar, since this is a substantial and evident problem at the present time worldwide, this problem is nutritional deficiency. It is also analyzed that this social metabolism can help to find a solution from the local to the regional and even the global, complementing the two concepts for the benefit of society. The type of methodology that was used consisted of a systematic review of updated information in academic articles on Food Sovereignty and Local Development, which was consulted in specialized databases such as Scopus and Web of Science

KEYWORDS: common goods, local development, sustainable development, territorial development, food sovereignty.

\section{Introducción}

El derecho a la alimentación es un derecho humano innato de la población mundial; este derecho se refleja en el organismo internacional de Naciones Unidas, que desarrolló la meta lc del Primer Objetivo de Desarrollo del Milenio, y esta meta se vuelve a establecer en el Segundo Objetivo de Desarrollo Sostenible para 2030, denominado "Hambre cero" (ONU, 2019). De igual manera, una dependencia de este organismo es la Organización para la Alimentación y la Agricultura, creada en 1945 con la finalidad de tratar los problemas urgentes de la alimentación mundial (FAO, 2020), la que a su vez estructura en 1974 el Comité de Seguridad Alimentaria Mundial (CSA), implementando el concepto de seguridad alimentaria (McMichael, 2009), pero a pesar de las metas propuestas el mundo se enfrenta a un número creciente de personas que carecen de alimentos suficientes para comer (Parra Contreras, 2020). Las cifras estimadas van desde el 2017 con 811 millones, al 2018 con 820 millones; estas cifras conllevan a una prevalencia de desnutrición de 10.8\% (FAO, 2019).

Aunque el discurso hegemónico justifica visiblemente la relevancia de luchar contra el hambre, la práctica muestra que aún faltan medidas concretas y parece que algunas estrategias simplemente han fallado (Dunford, 2020). Bajo este argumento nacen enfoques alternativos que amplían el concepto de seguridad alimentaria y derecho a la alimentación, 
REVISTA DE LA UNIVERSIDAD DEL ZULIA. 3ª época. Año 12 N³2, 2021 Arnaldo Vergara-Romero et al. //La soberanía alimentaria en el desarrollo local, 54-69 DOI: http://dx.doi.org/10.46925//rdluz.32.05

conduciendo a una nueva idea política: la Soberanía alimentaria (Patel, 2009). Con este concepto, los derechos de los productores de alimentos a la autodeterminación de su dieta y las técnicas culturales de cultivo agrícola, se tienen en cuenta y se fortalecen por primera vez (Bezner Kerr, Hickey, Lupafya, \& Dakishoni, 2019).

A partir de este nuevo concepto nacen muchos movimientos que se esfuerzan por realizar cambios en diferentes escalas territoriales y viendo el problema desde una perspectiva local (Ebel, et al., 2020). Dicha perspectiva orienta un Desarrollo local, provocando efectos sobre la sostenibilidad social, ecológica y económica dentro de estas comunidades (Beingessner \& Fletcher, 2020).

En este artículo se muestra al concepto de Soberanía alimentaria como mecanismo para el Desarrollo local, y cómo este proporciona la base para el estudio de los efectos desde lo local hacia un marco internacional global.

\section{Metodología}

El tipo de metodología que se utilizó consistió en una revisión sistemática de información actualizada en artículos académicos sobre Soberanía alimentaria y Desarrollo local, la cual fue consultada en bases especializadas como Scopus y Web of Science, en los periodos comprendidos desde el 2014 hasta el 2020, con búsqueda en título, resumen, palabras claves y en algunos casos el keywords plus de WOS.

Se utilizó el método histórico, ya que se empleó para determinar la evolución del concepto de Soberanía alimentaria, colocando este concepto a la actualidad y posturas modernas sobre el tema que se quiere abordar. Para ello, se utilizó la búsqueda de los términos "food sovereignty", "food AND sovereignty", "food sovereignty AND local development" y “food sovereignty AND local economy”. De igual manera se utilizó el método lógico sistémico que suministra la orientación general para dar cumplimiento al objetivo de artículo, a partir de la realización de un análisis integral de la Soberanía alimentaria.

\section{Revisión teórica}

El movimiento global por la Soberanía alimentaria está trabajando en una democratización fundamental del sistema agrícola y alimentario, así como en la reapropiación de bienes comunes. Es de importancia cuestionarse: ¿Qué contribución puede aportar el 
REVISTA DE LA UNIVERSIDAD DEL ZULIA. 3ª época. Año 12 N 32, 2021 Arnaldo Vergara-Romero et al. //La soberanía alimentaria en el desarrollo local, 54-69 DOI: http://dx.doi.org/10.46925//rdluz.32.05

concepto de Soberanía alimentaria a la participación de personas de todos los grupos sociales a nivel de desarrollo local?

La respuesta a esta pregunta se la puede encontrar en la Declaración Nyeleni en Sélingué-Malí, con su replicación en el Primer Foro Europeo para la Soberanía alimentaria, que menciona:

Nos oponemos y luchamos contra la mercantilización, la financiación y la patentización de nuestros bienes comunes, como: la tierra, las semillas tradicionales y reproducibles de campesinos y campesinas, las razas de ganado y bancos pesqueros; los árboles y los bosques; el agua, la atmosfera y el conocimiento. El acceso a estos bienes no debe de estar determinado por los mercados o por el capital. A la hora de utilizar estos recursos comunes debemos garantizar el respeto de los derechos humanos, el equilibrio de género, el beneficio del conjunto de la sociedad y el respeto de los derechos de la madre tierra. Nuestros bienes comunes deben ser manejados a través de la gestión colectiva, democrática y del control comunitario (LVC, 2011).

En los últimos años se ha hablado cada vez más de la crisis alimentaria como un problema integrado de múltiples desafíos. Esta crisis, que solo puede entenderse en interacción con muchas otras crisis se manifiesta por un lado en el hecho de que alrededor de 900 millones de personas en el mundo mueren de hambre y no solo en países en desarrollo y en vías de desarrollo (Schwab do Nascimento, Calle-Collado, \& Muñoz Benito, 2020). Entre las otras crisis, se puede mencionar la crisis del mercado financiero, energética, climáticos, materias primas, democráticas, sanitarias, etc.

Por otro lado, se manifiesta que en los países desarrollados y/o industrializados, cada vez más personas no tienen acceso a alimentos de alta calidad, culturalmente adaptados y variados (Naylor, 2019). Es significativo exponer que la pobreza alimentaria en los Estados Unidos y la Unión Europea, también se extiende contribuyendo al problema del hambre en el mundo, mediante una expresión de un alto interés en las ganancias del sistema agrícola y alimentario que se alinean en estas dos zonas geográficas (Noll \& Murdock, 2020).

Esta estrategia alineada se lleva a cabo mediante la exportación de productos claves para el sistema agrícola y alimentario hacia países del Sur global, reportando resultados de caída de los salarios, aumento de desempleo y la erosión de los sistemas de bienestar (OterosRozas, Ruiz-Almeida, Aguado, González, \& Rivera-Ferre, 2019). 
REVISTA DE LA UNIVERSIDAD DEL ZULIA. 3ª época. Año 12 N 32, 2021 Arnaldo Vergara-Romero et al. //La soberanía alimentaria en el desarrollo local, 54-69 DOI: http://dx.doi.org/10.46925//rdluz.32.05

Es claro que la industria alimentaria es uno de los pocos sectores que, a pesar de la "Gran Recesión" del 2008, puede esperar un aumento de las tasas de beneficio. Sus productos se basan en materias primas baratas, porque están subsidiadas en varios países, se venden a precios altos y la mayoría de consumidores enferman debido a su alto contenido de grasa y azúcar (Naylor, 2020).

Esto se basa en el pensamiento natural de que todos tenemos que comer, pero son pocas las personas que se preocupan de donde viene los alimentos, quién los produce y en qué circunstancias, qué luchas van de la mano con su distribución. El acceso a la tierra y otros bienes necesarios para la producción de alimentos, como semillas o agua, es un terreno muy disputado a nivel mundial.

La agricultura en su forma agroindustrial también crea muchos problemas según Mckay (2019), por ejemplo:

- La agroindustria es el mayor productor de CO2 y responsable de la degradación de muchos suelos.

- Los químicos que se usa para producir cada vez más alimentos inferiores contaminan el agua, el aire y el suelo.

- La ingeniería genética, los sistemas de producción controlados por computadora, los mataderos, plantas y animales se degrada a factores de producción.

Los diversos problemas de la agroindustria no son nada nuevo, pero es solo desde la crisis de precios de los alimentos de 2008 que existe un número creciente de investigadores comienzan a percibirlo como un problema coyuntural (Andrade Júnior, 2020).

\section{Soberanía alimentaria}

El concepto de Soberanía alimentaria fue introducido en 1996 en la Cumbre Mundial de la Organización de las Naciones Unidas para la Agricultura y la Alimentación (FAO) por la Vía campesina, la alianza global de pequeños agricultores, trabajadores agrícolas, pescadores, personas sin tierras e indígenas (Altieri \& Toledo, 2011).

Desde entonces, se ha convertido en el levantamiento político rector para la dirección de un número creciente de actores sociales de una amplia variedad de sectores sociales: movimientos, iniciativas y organizaciones del sector agrícola, de la escena de protección 
REVISTA DE LA UNIVERSIDAD DEL ZULIA. 3ª época. Año 12 N 32, 2021 Arnaldo Vergara-Romero et al. //La soberanía alimentaria en el desarrollo local, 54-69 DOI: http://dx.doi.org/10.46925//rdluz.32.05

ambiental, organizaciones de derechos humanos, consumidores y movimientos de género, pero en la actualidad también se agrupan movimientos urbanos en todo el mundo; todas estas agrupaciones tienen un marco común que es un cambio fundamental en el sistema agrícola y alimentario (Siebert, 2020).

El concepto de la Soberanía alimentaria no es un modelo terminado, sino que es un modelo que se adapta continuamente a los desafíos sociales, económicos y espaciales respectivos, y se desarrolla aún más democráticamente. Tiene su origen en la crítica del concepto técnico de seguridad alimentaria utilizado por instituciones como la FAO o el Banco mundial. El término Seguridad alimentaria se refiere a la disponibilidad y posiblemente a la calidad de los alimentos, incluyendo el acceso de la población a ellos (Noll \& Murdock, 2020).

Sin embargo, el término de Seguridad alimentaria no dice nada acerca de cómo y por quién se producen estos alimentos, quién los distribuye y consume. De este modo, oculta las condiciones de producción, el poder y las relaciones de gobierno, lo que puede llevar a políticas públicas de agricultura basada en monocultivos y ganadería industria, que a su vez contamina el suelo, el agua, daña el clima y necesita a los migrantes como trabajadores mal pagados y precarios; por lo tanto, la Seguridad alimentaria no garantiza el uso sostenible basado en el ciclo y cuidados de los recursos de forma constructiva de la agricultura (Pendergrast, Smith, Liebert, \& Bezner Kerr, 2019).

En contraste, la Soberanía alimentaria es el derecho de todas las personas a alimentos buenos y culturalmente adaptados que se producen utilizando métodos de producción sostenible, así como el derecho de las personas, las naciones y las comunidades internacionales a determinar su propia política alimentaria y agrícola (Dale, 2020).

La Soberanía alimentaria se basa en el establecimiento de sistemas de producción locales o regionales que están conectados en red de diversas maneras, el fortalecimiento del control local, la participación y la solidaridad internacional; por lo tanto, en una profunda democratización de las condiciones sociales, ecológicas y económicas que dan forma al sistema agrícola y alimentario (Rivera-Núñez, Fargher, \& Nigh, 2020).

\section{Desarrollo local}


REVISTA DE LA UNIVERSIDAD DEL ZULIA. 3ª época. Año 12 N 32, 2021 Arnaldo Vergara-Romero et al. //La soberanía alimentaria en el desarrollo local, 54-69 DOI: http://dx.doi.org/10.46925//rdluz.32.05

El Desarrollo local incorpora una habilidad diferente para el desarrollo en la sociedad global. Sorhegui-Ortega, expone un concepto amplio de Desarrollo local como:

El proceso reactivo de la economía y dinamizador de la sociedad local, que mediante el aprovechamiento de recursos endógenos existentes en una determinada zona o espacio físico es capaz de estimular y fomentar su crecimiento económico, crear empleo, renta, riqueza y sobre todo, mejorar la calidad de vida y el bienestar social de la comunidad (Sorhegui-Ortega, 2015).

La teoría del desarrollo local supone una expansión del concepto clásico de desarrollo, tomando en cuenta aspectos económicos, ecológicos, socioculturales y políticos. Consecuentemente, es crucial que tanto la población como los tomadores de decisiones identificarse con la región como su área viva y económica, por lo tanto, existe una conciencia regional (León-Segura \& Sorhegui-Ortega, 2019).

Es importante definir que el núcleo de la estrategia de desarrollo local es reconocer el potencial subutilizado y luego activarlo. Según Foissner (2000), se deben identificar esos potenciales locales con los siguientes criterios, tal como se muestra en la figura l:

El criterio del cuello de botella se basa en la diferente escasez de los potenciales individuales, de modo que los potenciales utilizados limitan las actividades locales y deben ser sustituidos por otros; un ejemplo es la capacidad de alimentación y nutrición.

El criterio de talento tiene como objetivo identificar los potenciales locales que compiten en la competencia regional.

Finalmente, el criterio de integración es llevar los potenciales a los ciclos regionales para lograr efectos de sinergia, porque la restricción de uno de estos criterios puede tener consecuencias indeseables. Los tres criterios deben usarse para mantener abierta las opciones de desarrollo regional.

5. Los bienes comunes entre la Soberanía alimentaria y el Desarrollo local

La variedad de cultivos agrícolas y ganaderos, los métodos de cultivo y las tecnologías para la producción de alimentos se han desarrollado, utilizado, conservado y adaptado a lo largo de miles de años. Fue y es considerado por muchos productores como bien común o bienes comunes, como base para que toda la agricultura sea compartida, administrada y protegida. 
REVISTA DE LA UNIVERSIDAD DEL ZULIA. 3ª época. Año 12 N 32, 2021 Arnaldo Vergara-Romero et al. //La soberanía alimentaria en el desarrollo local, 54-69

En un proceso históricamente único, estos recursos y su conocimiento subyacente fueron y son expropiados para la producción de alimentos capitalista. Las patentes sobre la vida, los reglamentos sobre la legislación de semillas en interés de las multinacionales agrícolas y la apropiación de las reservas de agua son solo algunos ejemplos de cómo organiza la exclusión sistemática que se dan en localidades de pequeña escala, afectando su desarrollo común (Mamonova \& Franquesa, 2019).

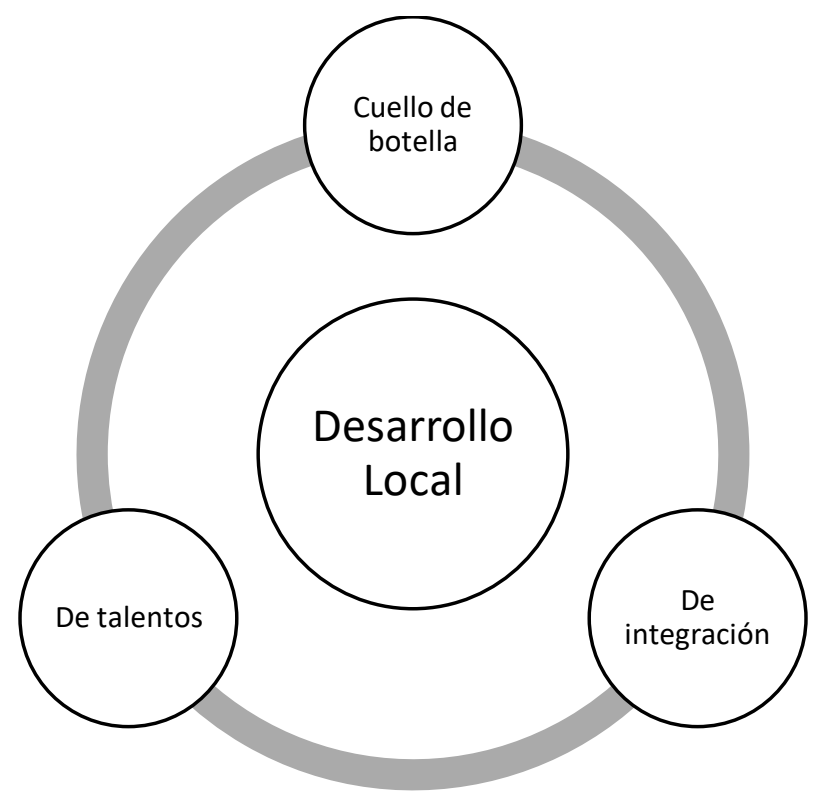

Figura 1. Criterios de potencialidades de Desarrollo local. Tomado de Foisner (2000).

A través de la mercantilización, la privatización y la monopolización del acceso a los "bienes comunes", este se regula por los mercados y el dinero, en lugar de ser objeto de negociaciones democráticas y procesos de diseño. Además, las corporaciones toman el control de lo que probablemente sea el bien común más importante en el campo de la producción de alimentos: el suelo. El acaparamiento de tierras está impulsado, entre otras cosas, por las políticas comerciales, energéticas y climáticas de los países más desarrollados y estos sirven a los intereses lucrativos del agronegocio y del capital financiero en busca de oportunidades de inversión (Stella, et al., 2019).

Se toma el ejemplo de las semillas, ya que es ideal para ilustrar los mecanismos de mercantilización y apropiación de bienes comunes. La semilla es la base de toda la diversidad 
REVISTA DE LA UNIVERSIDAD DEL ZULIA. 3ª época. Año 12 N 32, 2021 Arnaldo Vergara-Romero et al. //La soberanía alimentaria en el desarrollo local, 54-69 DOI: http://dx.doi.org/10.46925//rdluz.32.05

agrícola y fue un bien común durante miles de años. En la actualidad, las semillas también son un producto importante que está sujeto a numerosas regulaciones nacionales e internacionales. Y, por último, pero no menos importante, las semillas son objeto de deseo de algunas multinacionales (Ruiz-Almeida \& Rivera-Ferre, 2019).

El deseo de las multinacionales sobre las semillas es debido a que la mayoría de las semillas utilizadas en la agricultura industrializada y orientada al mercado deben comprarse a un precio costoso. Se están desarrollando nuevas variedades híbridas con propiedades aún mejores en laboratorios de alta tecnología, pero los agricultores no pueden influir en las características de variedad deseadas, ni se les permite replicar semillas (Martínez-Torres \& Rosset, 2010).

Además, la industria agroquímica intenta influir en las regulaciones de la legislación sobre semillas en beneficio a los intereses de dichas corporaciones, por ejemplo, la regulación de semillas negociada actualmente por las naciones en el mundo amenaza con restringir aún más los derechos de los agricultores para comerciar y vender semillas, al tiempo que fortalece los intereses corporativos (Bilewicz, 2020).

Sin embargo, las corporaciones multinacionales hacen más que solo controlar las semillas, estas corporaciones practican el mecanismo de patentar la propiedad intelectual de los métodos de mejoramiento genético y, por lo tanto, establecen una red difícil de entender en los sistemas internacionales de comercio y licencias (McMichael, 2009).

Este comercio de propiedad intelectual vulnera la agricultura tradicional, al igual que el compartir y controlar el propio conocimiento o conocimiento ancestral. Sin embargo, las semillas son la base de la subsistencia de la población, particularmente en numerosos países de África y Latinoamérica. Las regulaciones y el patentado privan a los agricultores de sus bases de producción y les niegan a los ciudadanos el acceso a alimentos variados y producidos de forma local (Levkoe, Brem-Wilson, \& Anderson, 2019).

Los movimientos sociales que defienden la Soberanía alimentaria son conscientes de que su lucha solo puede tener éxito si reclaman sus bienes comunes. Principalmente si se trata de acceso seguro y no discriminatorio, así como el control y la preservación sostenible de dichos bienes.

6. Una buena alimentación para el Desarrollo local 
REVISTA DE LA UNIVERSIDAD DEL ZULIA. 3ª época. Año 12 N 32, 2021 Arnaldo Vergara-Romero et al. //La soberanía alimentaria en el desarrollo local, 54-69 DOI: http://dx.doi.org/10.46925//rdluz.32.05

Es un hecho bien conocido que el hambre y la desnutrición no se basan en problemas de producción, sino en problemas de acceso y esto a su vez trae conflictos en el Desarrollo local. El acceso a los alimentos se organiza en el sistema capitalista mediante el control de la tierra y otros recursos productivos o mediante el mercado, es decir, a través de las relaciones de intercambio. Las personas que padecen hambre en ocasiones no tienen recursos productivos o dinero, por lo tanto, se pueden considerar personas en extrema pobreza y no pueden pagar por suficientes alimentos para su desarrollo personal (Borras, 2020).

Pero, ¿Qué pasa con los países industrializados? ¿Qué pasa con sus sistemas de producción de alimentos altamente diferenciados y de división de trabajo? ¿Puede la reconquista de los bienes comunes ayudar a combatir la pobreza? ¿Son los diversos proyectos alternativos relacionados con la alimentación y su producción un componente básico para una sociedad más justa e igualitaria?

Las iniciativas y movimientos que desean establecer y anclar la Soberanía alimentaria como una práctica cotidiana están trabajando para democratizar la forma en que se producen, fabrican y distribuyen los alimentos. Se trata de asumir la responsabilidad del estilo de vida, de las generaciones futuras y el estado actual del sistema agrícola y alimentario a nivel de países desarrollados o en vías de desarrollo (Gordon, 2020).

El núcleo del concepto de Soberanía alimentaria es el derecho de los actores, es decir, todos los que producen, procesan, distribuyen y comen alimentos, a negociar democráticamente el diseño del sistema. Para poder ejercer este derecho a la inclusión y participación, se necesitan requisitos previos que no son una cuestión habitual en nuestra sociedad, que se caracteriza por mecanismos de exclusión y relaciones de poder. Las personas de bajos ingresos y/o en extrema pobreza en particular a menudo están excluidas de la participación, por lo tanto, la Soberanía alimentaria quiere y debe luchar por condiciones que permitan a todos reclamar sus derechos (Baker-Médard \& Faber, 2020).

El derecho a tener derechos es el principio rector y el prerrequisito básico para la democratización de nuestro sistema agrícola y alimentario, por lo tanto, la Soberanía alimentaria es, ante todo, una emancipación, un modelo social basado en la solidaridad entre ellos, que tiene como objetivo realinear democráticamente la economía a las necesidades reales de las personas y transformar los bienes expropiados, privatizados y comercializados 
REVISTA DE LA UNIVERSIDAD DEL ZULIA. 3ª época. Año 12 N 32, 2021 Arnaldo Vergara-Romero et al. //La soberanía alimentaria en el desarrollo local, 54-69 DOI: http://dx.doi.org/10.46925//rdluz.32.05

en bienes comunes, parta usarlos y administrarlos de una manera democrática de base igualitaria (Levidow, Sansolo, \& Schiavinatto, 2019).

\section{7. ¿Y su aplicación?}

Un ejemplo destacado de resistencia, organización y solidaridad en el sentido de la Soberanía alimentaria se puede observar en la reciente crisis en Grecia. En muchos casos, la población que sufre políticas de austeridad ya no puede obtener alimentos básicos. El precio de la papa se elevó en tiempos de crisis, llevando los márgenes de beneficio a los intermediarios, mientras que los productores seguían recibiendo valores bien bajos por su producto. Bajo este comportamiento de mercado en tiempo de crisis, el campesinado griego funda el "movimiento de papas".

En primer lugar, los agricultores distribuían papas a la población de forma gratuita, después se fundaron las primeras plataformas de marketing directo para vender papas, pero luego también se anexaron otros alimentos básicos, dando así un nuevo mercado que se vende directamente al ciudadano y eliminar el comercio intermedio de los grupos de productores.

En la actualidad, el movimiento "sin intermediarios" (CHORIS MESAZONTES, en griego) organiza los mercados de agricultores en numerosas ciudades griegas. No solo se trata de productos asequibles para los consumidores finales y los precios al productor que cubren los costos, sino también de solidaridad e iniciativa. De igual manera, se organizan divisiones organizativas a pequeña escala que organizan ventas sin comercio intermedio y están comprometidos con la privatización del agua o las cargas tributarias inapropiadas, incluyendo las necesidades de las personas particularmente afectadas por la pobreza. Por ejemplo, los comestibles que no se vendieron en los mercados de agricultores se distribuyen a los necesitados de forma gratuita por la noche (Matacena \& Corvo, 2019).

El concepto de la Soberanía alimentaria está creciendo y progresando no solo en los países afectados por la "Gran recesión", donde las políticas de austeridad han tenido consecuencias dramáticas para la población (Beingessner \& Fletcher, 2020). Existen prácticas de Soberanía alimentaria a nivel local, regional y mundial: jardines interculturales, mercados de intercambio de plantas, cooperativas de alimentos, patios comunitarios, proyectos de agricultura urbana, cooperación de agricultores, plantación comunitaria, etc. En 
REVISTA DE LA UNIVERSIDAD DEL ZULIA. 3ª época. Año 12 N 32, 2021 Arnaldo Vergara-Romero et al. //La soberanía alimentaria en el desarrollo local, 54-69 DOI: http://dx.doi.org/10.46925//rdluz.32.05

todas las iniciativas y movimientos expuesto, las personas trabajan para conquistar la producción y distribución de alimentos (Levkoe, Brem-Wilson, \& Anderson, 2019).

En la actualidad, debido al número y/o tamaño, estas iniciativas y movimientos aun no pueden detener el sistema agrícola y alimentario vigente y sus mecanismos de exclusión y represión. A pesar de sus aspiraciones, hasta ahora no han logrado incluir a las personas que tienen que vivir en la pobreza en un grado insuficiente. Todavía se necesita un cierto esfuerzo y procesos de aprendizaje comunes para lograr la participación de personas de todos los grupos sociales y para abrir un alcance autónomo para la acción, incluso para los excluidos (Ho, 2020).

\section{Conclusiones}

La necesidad de implementar alternativas de pensamientos heterodoxos sobre alimentación y que, a su vez, beneficie al desarrollo local es muy evidente en las páginas anteriores, que dan pauta al marco contextual que se vive en la actualidad.

El devenir histórico sobre la alimentación, lleva a reflexionar que el buen uso de los bienes comunes refuerza al progreso del concepto de Soberanía alimentaria. Este progreso es un factor muy importante que ayuda al desarrollo local, ya que sin alimentación no podría surgir un desarrollo pleno, pues el hambre entra en un círculo vicioso entre la salud y la productividad.

Es significativo concluir que para complementar el metabolismo social todavía falta más esfuerzo de los colectivos y/o territorios a pequeña escala y que se necesita poner a disposición experiencias, practicar intercambios, mostrar solidaridad, compromiso y creatividad, estos son requisitos esenciales para un movimiento que apunta al bienestar de la sociedad y el desarrollo local.

\section{Recomendaciones}

Para futuras investigaciones se recomienda:

-Un análisis multivariante exploratorio sobre las dimensiones del concepto de Soberanía alimentaria, para revisar los componentes principales que tengan una relación con la variable latente de investigación, para luego realizar investigaciones confirmatorias. 
REVISTA DE LA UNIVERSIDAD DEL ZULIA. 3ª época. Año 12 N 32, 2021 Arnaldo Vergara-Romero et al. //La soberanía alimentaria en el desarrollo local, 54-69 DOI: http://dx.doi.org/10.46925//rdluz.32.05

-Un análisis correlacional entre la latente de investigación (Soberanía alimentaria) y el Valor Agregado Bruto (VAB) territorial, para analizar el contexto del crecimiento económico y los mecanismos agroecológicos.

-Realizar un análisis fenomenológico sobre la incidencia de cada país latinoamericano que tenga inmersa mediante ley el concepto de Soberanía alimentaria.

\section{Referencias}

Altieri, M. A., \& Toledo, V. M. (2011). The agroecological revolution in Latin America: Rescuing nature, ensuring food sovereignty and empowering peasants. Journal of Peasant Studies, 38(3), 587-612. https://doi.org/10.1080/03066150.2011.582947.

Andrade Júnior, H. d. (2020). Combating global hunger and forging a path to food sovereignty. Cadernos de Saúde Pública, 36(1), 1-3. https://doi.org/10.1590/0102$311 \times 00208719$.

Baker-Médard, M., \& Faber, J. (2020). Fins and (Mis)fortunes: Managing shark populations for sustainability and food sovereignty. Marine Policy, 113, https://doi.org/10.1016/j.marpol.2019.103805.

Beingessner, N., \& Fletcher, A. J. (2020). "Going local": farmers' perspectives on local food systems in rural Canada. Agriculture and Human Values, 37(1), 129-145. https://doi.org/10.1007/s10460-019-09975-6.

Bezner Kerr, R., Hickey, C., Lupafya, E., \& Dakishoni, L. (2019). Repairing rifts or reproducing inequalities? Agroecology, food sovereignty, and gender justice in Malawi. Journal of Peasant Studies, 46(7), 1499-1518. https://doi.org/10.1080/03066150.2018.1547897.

Bilewicz, A. M. (2020). Beyond the Modernisation Paradigm: Elements of a Food Sovereignty Discourse in Farmer Protest Movements and Alternative Food Networks in Poland. Sociologia Ruralis, 1-19. https://doi.org/10.1111/soru.12295.

Borras, S. M. (2020). Agrarian social movements: The absurdly difficult but not impossible agenda of defeating right-wing populism and exploring a socialist future. Journal of Agrarian Change, 20(1), 3-36. https://doi.org/10.1111/joac.12311.

Dale, B. (2020). Alliances for agroecology: from climate change to food system change. Agroecology and Sustainable Food Systems, 44(5), 629-652. https://doi.org/10.1080/21683565.2019.1697787.

Dunford, R. (2020). Converging on food sovereignty: transnational peasant activism, pluriversality and counter-hegemony. Globalizations, $0(7)$, 1-15. https://doi.org/10.1080/14747731.2020.1722494. 
REVISTA DE LA UNIVERSIDAD DEL ZULIA. $3^{a}$ época. Año $12 \mathrm{~N}^{\circ}$ 32, 2021 Arnaldo Vergara-Romero et al. //La soberanía alimentaria en el desarrollo local, 54-69 DOI: http://dx.doi.org/10.46925//rdluz.32.05

Ebel, R., Fallahi, E., Griffis, J. L., Nandwani, D., Nolan, D., Penhallegon, R. H., \& Rogers, M. (2020). Urban horticulture, from local initiatives to global success stories. Hort Technology, 30(1), 4-5. https://doi.org/10.21273/HORTTECH04525-19.

FAO. (2020). Food and Agriculture Organization. Recuperado el 02 de noviembre de 2020, de http://www.fao.org/about/es/

FAO. (2019). Food and Agriculture Organization. Recuperado el 05 de abril de 2019, de http://www.fao.org: http://www.fao.org/hunger/es/

Foissner, P. (2000). Endogene Entwicklung in peripheren Regionen : Möglichkeiten der Aktivierung endogener Potenziale in der Region Vorpommern. Raumforschung und Raumordnung, 58(4), 297-306. https://doi.org/10.1007/BF03183795.

Gordon, R. (2020). Productive Paradoxes: Exploring Prefigurative Practices with Derrida through a Spanish Food Sovereignty Collective. Antipode, 1-17. https://doi.org/10.1111/anti.12619.

Ho, H. T. (2020). Cosmopolitan locavorism: global local-food movements in postcolonial Hong Kong. Food, Culture and Society, 23(2), 137-154. https://oi.org/10.1080/15528014.2019.1682886.

León-Segura, C. M., \& Sorhegui-Ortega, R. (2019). El Desarrollo Local en la Globalización. Revista Economía y Desarrollo, 138(2).

Levidow, L., Sansolo, D., \& Schiavinatto, M. (2019). Agroecological practices as territorial development: an analytical schema from Brazilian case studies. Journal of Peasant Studies, 126. https://doi.org/10.1080/03066150.2019.1683003.

Levkoe, C. Z., Brem-Wilson, J., \& Anderson, C. R. (2019). People, power, change: three pillars of a food sovereignty research praxis. Journal of Peasant Studies, 46(7), 1389-1412. https://doi.org/10.1080/03066150.2018.1512488.

LVC. (2011). La Vía Campesina. Recuperado el 05 de abril de 2020, de https:/viacampesina.org/: https:/viacampesina.org/es/declaracion-nyeleni-europa-por-lasoberania-alimentaria-en-europa-ya/

Mamonova, N., \& Franquesa, J. (2019). Populism, Neoliberalism and Agrarian Movements in Europe. Understanding Rural Support for Right-Wing Politics and Looking for Progressive Solutions. Sociologia Ruralis, https://doi.org/10.11ll/soru.12291.

Martínez-Torres, M., \& Rosset, P. M. (2010). La vía campesina: The birth and evolution of a transnational social movement. Journal of Peasant Studies, 37(1), 149-175. https://doi.org/10.1080/03066150903498804.

Matacena, R., \& Corvo, P. (2019). Practices of Food Sovereignty in Italy and England: Short Food Supply Chains and the Promise of De-Commodification. Sociologia Ruralis, https://doi.org/10.1111/soru.12283. 
REVISTA DE LA UNIVERSIDAD DEL ZULIA. $3^{a}$ época. Año $12 \mathrm{~N}^{\circ}$ 32, 2021 Arnaldo Vergara-Romero et al. //La soberanía alimentaria en el desarrollo local, 54-69 DOI: http://dx.doi.org/10.46925//rdluz.32.05

McKay, B. M. (2019). Food sovereignty and neo-extractivism: limits and possibilities of an alternative development model. Globalizations, 1-19. https://doi.org/10.1080/14747731.2019.1691798.

McMichael, P. (2009). A food regime genealogy. Journal of Peasant Studies, 36(1), 139-169. https://doi.org/10.1080/03066150902820354.

Naylor, L. (2019). Food sovereignty in place: Cuba and Spain. Agriculture and Human Values, 36(4), 705-717. https://doi.org/10.1007/s10460-019-09938-x.

Naylor, L. (2020). Geopolitics and Food Sovereignty: Cuban Imaginaries. Geopolitics, 1-24. https://doi.org/10.1080/14650045.2019.1707187.

Noll, S., \& Murdock, E. G. (2020). Whose Justice is it Anyway? Mitigating the Tensions Between Food Security and Food Sovereignty. Journal of Agricultural and Environmental Ethics, 33(1), https://doi.org/10.1007/s10806-019-09809-9.

Oteros-Rozas, E., Ruiz-Almeida, A., Aguado, M., González, J. A., \& Rivera-Ferre, M. G. (2019). A social-ecological analysis of the global agrifood system. Proceedings of the National Academy of Sciences of the United States of America, 116(52), 26465-26473. https://doi.org/10.1073/pnas.19l2710116.

ONU. (abril de 2019). Organización de Naciones Unidas. Recuperado el 05 de abril de 2019, de https://www.un.org: https://www.un.org/sustainabledevelopment/es/hunger/

Parra Contreras, R. (2020). Una perspectiva del mundo que se nos avecina, Revista de la Universidad del Zulia, 11 (29), 3-5. DOI: http://dx.doi.org/10.46925/rdluz.29.01

Patel, R. (2009). What does food sovereignty look like? Journal of Peasant Studies, 36(3), 663-706. https://doi.org/10.1080/03066150903143079.

Pendergrast, T. L., Smith, B. J., Liebert, J. A., \& Bezner Kerr, R. (2019). Introduction to the symposium: rethinking food system transformation-food sovereignty, agroecology, food justice, community action and scholarship. Agriculture and Human Values, 36(4), 819-823. https://doi.org/10.1007/s10460-019-09952-z.

Rivera-Núñez, T., Fargher, L., \& Nigh, R. (2020). Toward an Historical Agroecology: an academic approach in which time and space matter. Agroecology and Sustainable Food Systems, 1-37. https://doi.org/10.1080/21683565.2020.1719450.

Ruiz-Almeida, A., \& Rivera-Ferre, M. G. (2019). Internationally-based indicators to measure Agri-food systems sustainability using food sovereignty as a conceptual framework. Food Security, 11(6), 1321-1337. https://doi.org/10.1007/s12571-019-00964-5.

Schwab do Nascimento, F., Calle-Collado, Á., \& Muñoz Benito, R. (2020). Economía social y solidaria y agroecología en cooperativas de agricultura familiar en Brasil como forma de desarrollo de una agricultura sostenible. CIRIEC-España, revista de economía pública, social y cooperativa (98), 189. https://doi.org/10.7203/ciriec-e.98.14161. 
REVISTA DE LA UNIVERSIDAD DEL ZULIA. 3ª época. Año 12 N 32, 2021 Arnaldo Vergara-Romero et al. //La soberanía alimentaria en el desarrollo local, 54-69

DOI: http://dx.doi.org/10.46925//rdluz.32.05

Siebert, A. (2020). Transforming urban food systems in South Africa: unfolding food sovereignty in the city. Journal of Peasant Studies, 47(2), 401-419. https://doi.org/10.1080/03066150.2018.1543275.

Sorhegui-Ortega, R. (2015). ¿Es viable el desarrollo local en Cuba? En: http://roa.ult.edu.cu/handle/123456789/3235

Stella, G., Coli, R., Maurizi, A., Famiani, F., Castellini, C., Pauselli, M., ... Manconi, M. E. (2019). Towards a National Food Sovereignty Plan: Application of a new Decision Support System for food planning and governance. Land Use Policy, 89, 104216. https://doi.org/10.1016/j.landusepol.2019.104216. 